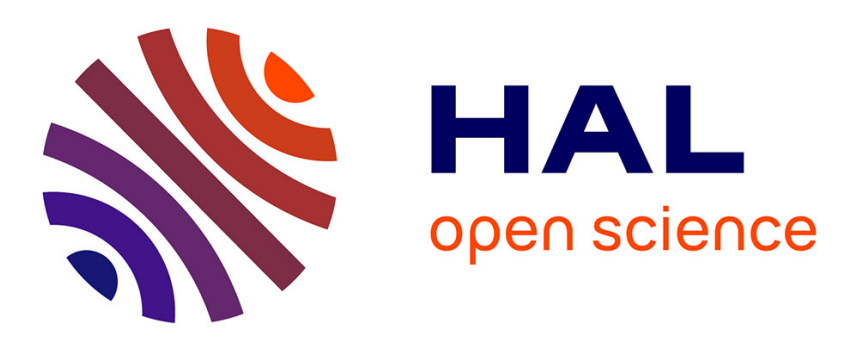

\title{
Integral Control Design using the Implicit Lyapunov Function Approach
}

\author{
Angel Mercado-Uribe, Jaime Moreno, Andrey Polyakov, Denis Efimov
}

\section{To cite this version:}

Angel Mercado-Uribe, Jaime Moreno, Andrey Polyakov, Denis Efimov. Integral Control Design using the Implicit Lyapunov Function Approach. 58th IEEE Conference on Decision and Control, Dec 2019,

Nice, France. hal-02371919

\section{HAL Id: hal-02371919 \\ https://hal.inria.fr/hal-02371919}

Submitted on 20 Nov 2019

HAL is a multi-disciplinary open access archive for the deposit and dissemination of scientific research documents, whether they are published or not. The documents may come from teaching and research institutions in France or abroad, or from public or private research centers.
L'archive ouverte pluridisciplinaire HAL, est destinée au dépôt et à la diffusion de documents scientifiques de niveau recherche, publiés ou non, émanant des établissements d'enseignement et de recherche français ou étrangers, des laboratoires publics ou privés. 


\title{
Integral Control Design using the Implicit Lyapunov Function Approach
}

\author{
Angel Mercado-Uribe, Jaime A. Moreno, Andrey Polyakov and Denis Efimov
}

\begin{abstract}
In this paper, we design homogeneous integral controllers of arbitrary non positive homogeneity degree for a system in the normal form with matched uncertainty/perturbation. The controllers are able to reach finitetime convergence, rejecting matched constant (Lipschitz, in the discontinuous case) perturbations. For the design, we use the Implicit Lyapunov Function method combined with an explicit Lyapunov function for the addition of the integral term.
\end{abstract}

\section{INTRODUCTION}

Consider a system that can be brought to the (ByrnesIsidori) normal form [1] which corresponds to

$$
\begin{aligned}
& \dot{\eta}=f_{0}(\eta, x, \rho(t)), \\
\dot{x}_{i}= & x_{i+1}, \quad i=1, \ldots, n-1, \\
\dot{x}_{n}= & f(\eta, x)+u+\rho, \\
y= & x_{1},
\end{aligned}
$$

where $\eta \in \mathbb{R}^{m}$ and $x \in \mathbb{R}^{n}$ are the states, $u \in \mathbb{R}$ is the control variable, $f(\eta, x)$ is assumed to be a known nominal non-linearity. Subsystem (1) represents the zero dynamics of the system, and $\rho$ corresponds to (matched) uncertainties/perturbations. If the zero dynamics is Input-to-State Stable (ISS) with respect to $x$ and $\rho$, then the robust tracking problem for the output $y$ can be reduced to the robust stabilization of the subsystem (2), rejecting the term $\rho$.

Note that if $\rho(t)$ is non-vanishing when $x$ is at the origin, system (2) cannot be stabilized by using a continuous static controller, and only "practical" stability can be attained. However, a memoryless discontinuous (at $x=0$ ) controller is able to compensate exactly the non-vanishing perturbation. (High Order) Sliding Mode Control [2], [3], [4] is a well known technique achieving this robust stabilization problem by means of discontinuous controllers. Homogeneous continuous or discontinuous controllers can be designed by different methods. In [5] the design is based on explicit Lyapunov functions, while in [6] the controllers are designed by means of an Implicitly defined Lyapunov Function. One attractive feature of this latter method is that the gain design is based on the selection of stabilizing gains for a linear system, and can be reduced to an LMI problem.

Angel Mercado-Uribe and Jaime A. Moreno are with Instituto de Ingeniería, Universidad Nacional Autónoma de México (UNAM), Coyoacán, D.F., 04510, México (e-mail: joseangelmu814@gmail.com, JMorenoPei i.unam.mx

A. Polyakov anf D. Efimov are with Inria, Univ. Lille, CNRS, UMR 9189 - CRIStAL, F-59000 Lille, France and ITMO University, 49 av. Kronverkskiy, 197101 Saint Petersburg, Russia (e-mail: andrey.polyakoveinria.fr, denis.efimoveinria.fr).
However, an undesirable effect of discontinuous controllers is the "Chattering" [3]. An alternative approach to compensate a non-vanishing perturbation $\rho$ is the use of dynamic controllers, being the integral control [7] the most classical one. However, in comparison to Sliding Mode Controllers, which can reject bounded perturbations, the classical integral controller is only able to compensate constant ones, which is by far a smaller class.

For systems with relative degree one a classical discontinuous integral controller is the well-known Super-Twisting Algorithm (see e.g. [8]). In [9] an explicit Lyapunov function has been proposed for the stability analysis and the gain design of the super twisting. Recently, nonlinear continuous and homogeneous integral controllers have been proposed for mechanical systems with relative degree two [10], but without a formal stability proof. A discontinuous and homogeneous integral controller has been proposed for systems with different relative degrees in [11], [12], [13], [14], [15], using some explicit Lyapunov functions for the design of the gains and the convergence and robustness proof. The basic idea of the construction of the (non-smooth) Lyapunov function for relative degree one used in [9] has been extended for arbitrary relative degree in [16] to obtain continuous and discontinuous integral controllers. All these methods present the drawback, that the gain selection becomes a highly nonlinear problem, making its application more difficult.

Since the design of (memoryless) homogeneous continuous or discontinuous feedback controllers using the Implicit Lyapunov Function (ILF) method [6] greatly simplifies the gain design and it can be systematized in contrast to other methods, we propose here to use it for the design of (continuous and discontinuous) integral controllers for system (2). Since a direct application of the ILF idea does not lead to a usable integral controller, we combine the ILF method for the design of a (rational) state feedback controller and an explicit Lyapunov function for the calculation of the integral part which resembles the idea used for the the SuperTwisting in [9], and which is generalized for arbitrary order in [16]. This leads to a very useful method for designing homogenous integral controllers of arbitrary non-positive degree, for which the gains design is greatly simplified. The continuous integral controllers are able to compensate constant perturbations (as in the classical integral action), while discontinuous controllers can reject Lipschitz perturbations. It is also worth noticing here, that since the control signal in the discontinuous integral action is obtained through the (time) integration of the signal produced by the discontinuous term, it is continuous and the effect of chattering can be in principle reduced. 


\section{A. Notation}

For a real variable $z \in \mathbb{R}$ and a real number $p \in \mathbb{R}$ the symbol $[z\rfloor^{p}=|z|^{p} \operatorname{sign}(z)$ is the signed power $p$ of $z$. According to this $\lceil z\rfloor^{0}=\operatorname{sign}(z)$. Note that $\lceil z\rfloor^{0}=\operatorname{sign}(z)$ is a multivalued function, i.e. $\lceil z\rfloor^{0}=1$ if $z>0,\lceil z\rfloor^{0} \in$ $[-1,+1]$ if $z=0,\lceil z\rfloor^{0}=-1$ if $z<0 . \frac{d}{d z}\lceil z\rfloor^{m}=m|z|^{m-1}$ and $\frac{d}{d z}|z|^{m}=m\lceil z\rfloor^{m-1}$. Note that $\lceil z\rfloor^{2}=|z|^{2} \operatorname{sign}(z) \neq z^{2}$, and if $p$ is an odd number then $\lceil z\rfloor^{p}=z^{p}$ and $|z|^{p}=z^{p}$ for any even integer $p$. Moreover $[z\rfloor^{p}\lceil z\rfloor^{q}=|z|^{p+q},\lceil z\rfloor^{p}\lceil z\rfloor^{0}=|z|^{p}$ and $\lceil z\rfloor^{0}\lceil z\rfloor^{p}=|z|^{p}$.

\section{PReliminaries}

For a given vector $x=\left(x_{1}, \ldots, x_{n}\right)^{T} \in \mathbb{R}^{n}$, the dilation operator is defined as $\Lambda_{r}(\varepsilon) x:=\left(\varepsilon^{r_{1}} x_{1}, \ldots, \varepsilon^{r_{n}} x_{n}\right), \forall \varepsilon>0$, where $r_{i}>0$ are the weights of the coordinates and $\mathbf{r}=\left(r_{1}, \ldots, r_{n}\right)$ is the vector of weights. A function $V: \mathbb{R}^{n} \rightarrow \mathbb{R}$ (respectively, a vector field $f: \mathbb{R}^{n} \rightarrow \mathbb{R}^{n}$, or a vector-set field $F(x) \subset \mathbb{R}^{n}$ ) is called r-homogeneous of degree $m \in \mathbb{R}$ if the identity $V\left(\Lambda_{r}(\varepsilon) x\right)=\varepsilon^{m} V(x)$ holds (resp., $f\left(\Lambda_{r}(\varepsilon) x\right)=\varepsilon^{m} \Lambda_{r}(\varepsilon) f(x)$, or $\left.F\left(\Lambda_{r}(\varepsilon) x\right)=\varepsilon^{m} \Lambda_{r}(\varepsilon) F(x)\right)$ [8], [17], [18]. A system is called homogeneous if its vector field (or vector-set field) is $\mathbf{r}$-homogeneous of some degree. The homogeneous norm is defined by $\|x\|_{\mathbf{r}, p}:=\left(\sum_{i=1}^{n}\left|x_{i}\right|^{\frac{p}{r_{i}}}\right)^{\frac{1}{p}}, \forall x \in \mathbb{R}^{n}$, for any $p \geq \max \left\{r_{i}\right\}$, and it is $\mathbf{r}$-homogeneous of degree 1 . The set $\mathbb{S}=\left\{x \in \mathbb{R}^{n}:\|x\|_{\mathbf{r}, p}=1\right\}$ is the corresponding unit sphere.

For a vector-set field $F(x)$ we denote by $L_{F} V(x)=$ $\left\{y \in \mathbb{R} \mid y=\partial_{x} V(x) \cdot v, v \in F(x)\right\}$ the set of values taken by Lie's derivatives of $V$ along all vector fields contained in $F(x)$. If $V$ and $F$ are $\mathbf{r}$-homogeneous, then so is $L_{F} V(x)$. Stability of homogeneous systems can be studied by means of homogeneous Lyapunov functions (HLFs) [19], [18], [20], [21], [22]. Any continuous homogeneous system $\dot{x}=f(x)$, with asymptotically stable (AS) equilibrium point, admits a $C^{p}$ HLF of degree $m$ if $m>p \cdot \max \left\{r_{i}\right\}$ for any $p \in \mathbb{N}$ [18]. A differential inclusion (DI) $-\dot{x} \in F(x)$ - is a (homogeneous) Filippov DI if $F(x)$ is a (homogeneous) vector-set field (multivalued map) satisfying standard assumptions, i.e. it is non-empty, closed, convex, locally bounded and uppersemicontinuous [23], [8]. When $0 \in F(x)$ the origin is an equilibrium point. It is strongly Globally Asymptotically Stable (GAS), i.e. all trajectories converge to the origin, iff there exists a $C^{\infty}$ (homogeneous) strong LF [20], [22], [17]. Moreover, if $F$ is r-homogeneous of degree $l<0$, then $x=0$ is strongly globally finite-time stable (GFTS) and the settling time is continuous at zero and locally bounded [8], [22], [17].

Finally, we add an important result about Implicitly defined Lyapunov Functions [6].

Theorem 1: Let the system

$$
\dot{x} \in f(t, x(t)), \quad t \in \mathbb{R}_{+}, \quad x(0)=x_{0} .
$$

If there exists a continuous function

$$
\begin{array}{r}
Q: \mathbb{R}_{+} \times \mathbb{R}^{n} \rightarrow \mathbb{R} \\
(V, x) \rightarrow Q(V, x)
\end{array}
$$

satisfying the conditions:
C1) $Q$ is continuously differentiable outside the origin;

C2) for any $x \in \mathbb{R}^{n} \backslash\{0\}$ there exists $V \in \mathbb{R}_{+}$such that

$$
Q(V, x)=0
$$

C3) let $\Omega=(V, x) \in \mathbb{R}_{+} \times \mathbb{R}^{n}: Q(V, x)=0$ and

$$
\lim _{x \rightarrow 0} V=0^{+}, \lim _{V \rightarrow 0^{+}}\|x\|=0, \lim _{\|x\| \rightarrow \infty} V=+\infty ; \quad \forall(V, x) \in \Omega
$$

C4) $\frac{\partial Q(V, x)}{\partial V}<0 \forall V \in \mathbb{R}_{+}$and $x \in \mathbb{R}^{n} \backslash\{0\}$;

C5) $\sup _{t \in \mathbb{R}_{+}, y \in f(t, x)} \frac{\partial Q(V, x)}{\partial x} y<0 \forall(V, x) \in \Omega$;

then the origin of system (3) is globally uniformly asymptotically stable.

Remark 1: It is important to stress that the existence of the function $Q$ in Theorem 1 implies that a Lyapunov function $V(x)$ exists. This can be seen by the required conditions. Condition $\mathrm{C} 2$ indicates that $V$ has to be positive definite for any $x \in \mathbb{R}^{n} \backslash\{0\}$. Condition C3 says that $V(0)=0$ and $V$ is radially unbounded, i. e. the stability is global. Finally, conditions $\mathrm{C} 4$ and C5 imply that $\dot{V}(x)$ is negative definite.

\section{Problem Statement}

In this paper, we consider a system as

$$
\dot{x}=A x+b(u+\rho)
$$

where $x \in \mathbb{R}^{n}$ is the state vector, $u \in \mathbb{R}$ is the control input and $\rho: \mathbb{R}_{+} \rightarrow \mathbb{R}$ is a perturbation. The matrix $A \in \mathbb{R}^{n \times n}$ is defined as $A=\left[\delta_{i, k}\right]$, where $\delta_{i, k}=1$ if $k=i+1$ and $\delta_{i, k}=0$ otherwise. Vector $b \in \mathbb{R}^{1 \times n}$ corresponds to $b^{T}=[0, \cdots, 0,1]$.

The control objective is to robustly stabilize the origin of system (4), asymptotically or in finite time, in spite of the perturbation $\rho$. In the absence of perturbation, i. e. $\rho(t) \equiv 0$, a memoryless continuous state feedback $u=\phi(x)$ can stabilize the origin of system (4). However, this is not feasible for a non vanishing perturbation, i.e. a static continuous feedback control cannot stabilize $x=0$. Therefore, the following dynamic control structure is proposed:

$$
u=u_{1}(x)+z, \quad \dot{z}=u_{2}(x),
$$

This controller consists of the state feedback $u_{1}(x)$, aimed at stabilizing the nominal system (i.e. without perturbation), and an integral term $z$, driven by the function $u_{2}(x)$, which must estimate and compensate the perturbation term.

We want to impose the condition that the closed loop system (without perturbation) is homogeneous, with non positive homogeneity degree. The weights of the variables $(x, z)$ are therefore $r_{i}=1+(i-1) v, i=1, \ldots, n+1$ and the vector of weights results in $\mathbf{r}=[1,1+v, \cdots, 1+(n-1) v, 1+n v]$, and the dilation matrix is $\Lambda_{r}(\lambda)=\operatorname{diag}\left\{\lambda^{r_{i}}\right\}$. We also define $r_{n+2}=1+(n+1) v$ for convenience. $v$ is the homogeneity degree, taking values in the interval $-(n+1)^{-1} \leq v \leq 0$ such that $r_{n+2} \geq 0$. Likewise, the controller functions $u_{1}(x)$ and $u_{2}(x)$ must be homogeneous functions of homogeneity degrees $r_{n+1}$ and $r_{n+2}$, respectively.

Note that for $v=0$, we recover a linear state feedback with integral control. However, on the other extreme, when $v=-(n+1)^{-1}$, function $u_{2}(x)$ has homogeneity degree zero, 
and therefore it is discontinuous, at least at the origin $x=0$. In this case the right hand side of the closed loop system is discontinuous, and the trajectories have to be understood in the sense of Filippov [23]. Note, that although $u_{2}$ is discontinuous, the control signal $u(t)$ is continuous, since it is the addition of a continuous state feedback $u_{1}(x)$ and the time integral of the discontinuous signal $u_{2}(x(t))$.

We want to find a method to design the controller functions $u_{1}(x)$ and $u_{2}(x)$ and characterize the class of perturbations for which the controlled system (4)-(5) renders $x=0$ asymptotically stable despite of any perturbation in the class.

\section{Main Result: Integral CONTROLleR DESIGN USING IMPLICIT LYAPUNOV FUNCTIONS}

In this section, we present the main result: a homogeneous integral controller (5) that robustly stabilizes the origin of system (4) despite arbitrary matched constant perturbations, when the homogeneity degree $v$ satisfies $-\frac{1}{n+1}<v \leq 0$, or Lipschitz continuous perturbations, when $v=-\frac{1}{n+1}$, i.e. when the integral controller $u_{2}(x)$ is discontinuous.

Theorem 2: Consider the system (4), with a matched Lipschitz continuous perturbation $\rho$, and fix a homogeneity degree $v \in\left[-\frac{1}{n+1}, 0\right]$, so that the weights $r_{i}=1+(i-1) v$, $i=1, \ldots, n+1$ are determined. Find a constant and symmetric matrix $P=P^{T} \in \mathbb{R}^{n \times n}$ and a vector $K \in \mathbb{R}^{n}$ such that for some $\varepsilon>0$ the following conditions are satisfied:

$$
\begin{aligned}
& H_{r} P+P H_{r}<0, \quad H_{r}=-\operatorname{diag}\left\{r_{i}\right\} \\
& P(A-b K)+(A-b K)^{T} P=\varepsilon\left(H_{r} P+P H_{r}\right), \quad \varepsilon>0 .
\end{aligned}
$$

Under these conditions the homogeneous integral controller

$$
\begin{aligned}
& u=-V^{1+n v} K \Lambda_{r}\left(V^{-1}\right) x+z \\
& \dot{z}=\gamma V^{(n+1) v}\left(Q_{V}\right)^{-1} b^{T} P \Lambda_{r}\left(V^{-1}\right) x, \quad \gamma>0
\end{aligned}
$$

where $V \in \mathbb{R}_{+}$is determined implicitely by the equation

$$
Q(V, x) \triangleq x^{T} \Lambda_{r}\left(V^{-1}\right) P \Lambda_{r}\left(V^{-1}\right) x-1=0,
$$

and

$$
Q_{V}=V^{-1} x^{T} \Lambda_{r}\left(V^{-1}\right)\left(H_{r} P+P H_{r}\right) \Lambda_{r}\left(V^{-1}\right) x,
$$

asymptotically stabilizes the origin of system (4)

1) exponentially despite any constant perturbation, i.e. $\dot{\rho}=0$, for any $\gamma>0$ if $v=0$;

2 ) in finite time despite any constant perturbation for any $\gamma>0$ if $-\frac{1}{n+1}<v<0$; or

3) in finite time and despite any Lipschitz perturbation with a Lipschitz constant $D \geq 0$, i.e. $|\dot{\rho}| \leq D$, for any $\gamma>0$ if $v=-\frac{1}{n+1}$. The value of $D$ depends on $\gamma$ and the other gains.

Moreover, the closed loop system is ISS with respect to $\rho$ if $-\frac{1}{n+1}<v \leq 0$.

Conditions (6) have always a solution $P$ and $K$ (see [6]), and they represent no restriction. The implicit equation (8) provides the value of $V$ for each $x=\left(x_{1}, \ldots, x_{n}\right)$, and in general has to be solved numerically on-line, since the value of $V$ is required for the implementation of the controller (7).
A numerical procedure is proposed in [6]. The controller gains $K$ are obtained from (6), which is relatively simple to solve numerically. The integral gain $\gamma>0$ can be in principle selected arbitrarily large, and the stability property is conserved. Only in the discontinuous case $\gamma$ affects the size $D$ of the class of Lipschitz perturbations that can be fully compensated. In general, the gain selection here is easier than for the integral controllers designed using explicit Lyapunov functions, as e.g. the ones presented in [24].

We note some differences between the controller (7) and those presented e.g. in [15], [14]: (i) In (7) the integral action $u_{2}$ depends on the full state $x$, while $u_{2}$ in [15], [14] can be a function of $x_{1}$ alone, or $x_{1}$ and a homogeneous function of any other states. (ii) For $v=-\frac{1}{n+1}$ the integral controller in (7) is discontinuous only at $x=0$, so that it is of the quasi-continuous form. However, $u_{2}$ in [15], [14] can be discontinuous on homogeneous varieties larger than the origin. (iii) For controller (7) the stability is assured for any arbitrary value of $\gamma>0$, while for the controllers in [15], [14] this is not always the case. (iv) The Lyapunov function (see next Section) for (7) is not smooth, but for the controllers in [15], [14] the Lyapunov functions are smooth. Moreover, the proof is completely different.

\section{Proof of Theorem 2: A Lyapunov Function APPROACH}

In this section, we will prove that Theorem 2 holds. In order to do it, we design first the (continuous) state feedback controller $u_{1}(x)$, using a Lyapunov Function obtained by the Implicit Lyapunov Function (ILF) approach proposed in [6]. Combining this ILF with another explicit term (borrowed from the construction of the Lyapunov Function for the Super-Twisting in [9] and extended for an arbitrary order in [16]) we obtain a (Strong) non smooth Control Lyapunov function for the closed loop system, from which we design the integral part of the controller $u_{2}(x)$ in (5).

Before presenting the procedures to design the controllers, we show that they are homogeneous. The homogeneity degree of $u_{1}(x)$ is $r_{n+1}=1+n v$ since

$$
\begin{aligned}
u_{1}\left(\Lambda_{r}(\lambda) x\right) & =-V^{1+n v}\left(\Lambda_{r}(\lambda) x\right) K \Lambda_{r}\left(V^{-1}\left(\Lambda_{r}(\lambda) x\right)\right) \Lambda_{r}(\lambda) x \\
& =-\lambda^{1+n v} V^{1+n v}(x) K \Lambda_{r}\left(V^{-1}(x)\right) x \\
& =\lambda^{1+n v} u_{1}(x) .
\end{aligned}
$$

Analogously, it is possible to prove that the homogeneity degree of $u_{2}(x)$ is $r_{n+2}=1+(n+1) v$.

\section{A. Design of the static feedback control $u_{1}(x)$ using an ILF}

First of all, we consider the reduced system

$$
\dot{x}=A x+b u_{1} \text {. }
$$

Based on [6], we propose the following quadratic function

$$
Q(V, x)=x^{T} \Lambda_{r}\left(V^{-1}\right) P \Lambda_{r}\left(V^{-1}\right) x-1, P=P^{T}>0 .
$$

The equation $Q(V, x)=0$ defines implicitly the homogeneous LF $V$ of homogeneity degree 1. 
Obtaining the derivative of $Q$

$$
\dot{Q}(V, x)=\frac{\partial Q(V, x)}{\partial V} \dot{V}+\frac{\partial Q(V, x)}{\partial x}\left(A x+b u_{1}\right)=0
$$

and so

$$
\dot{V}=-\left(Q_{V}(V, x)\right)^{-1} \frac{\partial Q(V, x)}{\partial x}\left(A x+b u_{1}\right),
$$

where

$$
\begin{aligned}
Q_{V}(V, x) & =\frac{\partial Q(V, x)}{\partial V} \\
& =V^{-1} x^{T} \Lambda_{r}\left(V^{-1}\right)\left(H_{r} P+P H_{r}\right) \Lambda_{r}\left(V^{-1}\right) x,
\end{aligned}
$$

where

$$
H_{r}=-\operatorname{diag}\left\{r_{i}\right\} .
$$

Note that in order to satisfy condition C4 in Theorem 1, the following condition has to hold

$$
H_{r} P+P H_{r}<0 .
$$

Likewise, we have that

$$
\frac{\partial Q(V, x)}{\partial x}=2 x^{T} \Lambda_{r}\left(V^{-1}\right) P \Lambda_{r}\left(V^{-1}\right) .
$$

Therefore, $\dot{V}$ can be written as follows

$$
\dot{V}=-\left(Q_{V}(V, x)\right)^{-1} 2 x^{T} \Lambda_{r}\left(V^{-1}\right) P \Lambda_{r}\left(V^{-1}\right)\left(A x+b u_{1}\right) \text {. }
$$

Using the following properties of $A$ and $b$

$$
\lambda^{v} \Lambda_{r}(\lambda) A=A \Lambda_{r}(\lambda), \quad \Lambda_{r}(\lambda) b=\lambda^{1+(n-1) v} b,
$$

we can write

$$
\begin{aligned}
\dot{V}= & -\left(Q_{V}(V, x)\right)^{-1} 2 x^{T} \Lambda_{r}\left(V^{-1}\right) P \times \\
& \left(V^{v} A \Lambda_{r}\left(V^{-1}\right) x+V^{-1-(n-1) v} b u_{1}\right) \\
= & -V^{v}\left(Q_{V}(V, x)\right)^{-1} x^{T} \Lambda_{r}\left(V^{-1}\right)\left(P A+A^{T} P\right) \Lambda_{r}\left(V^{-1}\right) x- \\
& 2 V^{-1-(n-1) v}\left(Q_{V}(V, x)\right)^{-1} x^{T} \Lambda_{r}\left(V^{-1}\right) P b u_{1} .
\end{aligned}
$$

Now, selecting

$$
u_{1}(x)=-V^{1+n v} K \Lambda_{r}\left(V^{-1}\right) x,
$$

which is homogeneous with homogeneity degree $1+n v$, we obtain

$$
\begin{aligned}
\dot{V}= & -V^{v}\left(Q_{V}(V, x)\right)^{-1} \times \\
& x^{T} \Lambda_{r}\left(V^{-1}\right)\left(P(A-b K)+(A-b K)^{T} P\right) \Lambda_{r}\left(V^{-1}\right) x .
\end{aligned}
$$

So, if we design $K$ such that ${ }^{1}$

$$
P(A-b K)+(A-b K)^{T} P=\varepsilon\left(H_{r} P+P H_{r}\right), \varepsilon>0,
$$

then

$$
\begin{aligned}
\dot{V} & =-\varepsilon V^{v}\left(Q_{V}(V, x)\right)^{-1} x^{T} \Lambda_{r}\left(V^{-1}\right)\left(H_{r} P+P H_{r}\right) \Lambda_{r}\left(V^{-1}\right) x \\
& =-\varepsilon V^{1+v},
\end{aligned}
$$

and thus $\dot{V}<0$.

\footnotetext{
${ }^{1}$ In the next expression we have changed the inequality by an equality to simplify some expressions.
}

B. Design of the dynamic control feedback $u_{2}(x)$ using an explicit (control) Lyapunov Function

Now, we add the integral term

$$
\dot{x}=A x+b\left(u_{1}+z\right), \quad \dot{z}=u_{2}+d
$$

where $d=\dot{\rho}(t) \leq D$. Note that the perturbation $\rho$ appearing in the input $u_{1}$ channel has been included in $z$, and moved down to the $u_{2}$ channel.

1) A weak Lyapunov Function: Consider the homogeneous of degree $2 r_{n+1}=2(1+n v)$ LF candidate

$$
W(x, z)=\frac{1}{2(1+n v)} V^{2(1+n v)}(x)+\frac{1}{\gamma} z^{2} .
$$

Its derivative is given by

$$
\begin{aligned}
\dot{W}= & V^{1+2 n v}\left\{-\varepsilon V^{1+v}-2 V^{-1-(n-1) v}\left(Q_{V}(V, x)\right)^{-1} \times\right. \\
& \left.x^{T} \Lambda_{r}\left(V^{-1}\right) P b z\right\}+\frac{2}{\gamma} z\left(u_{2}+d\right) \\
= & -\varepsilon V^{2(1+n v)+v}-2 V^{(n+1) v}\left(Q_{V}(V, x)\right)^{-1} \times \\
& x^{T} \Lambda_{r}\left(V^{-1}\right) P b z+\frac{2}{\gamma} z u_{2}+\frac{2}{\gamma} z d .
\end{aligned}
$$

Now, we select $u_{2}(x)$ such that

$$
-2 V^{(n+1) v}\left(Q_{V}(V, x)\right)^{-1} x^{T} \Lambda_{r}\left(V^{-1}\right) P b z+\frac{2}{\gamma} z u_{2}=0 .
$$

This implies that

$$
u_{2}(x)=\gamma V^{(n+1) v}\left(Q_{V}(V, x)\right)^{-1} b^{T} P \Lambda_{r}\left(V^{-1}\right) x,
$$

where $\gamma>0$ can be selected freely. In that case we have

$$
\dot{W}=-\varepsilon V^{2(1+n v)+v}+\frac{2}{\gamma} z d .
$$

When $d \equiv 0$ the function $\dot{W} \leq 0$ is negative semidefinite, and $W$ is a weak Lyapunov function. LaSalle's invariance theorem imply asymptotic stability. Homogeneity of degree zero implies exponential, while negative degree means finite time stability of the origin.

2) A strong Lyapunov function: Using the weak LF (12), we can add a cross term to obtain a Strong LF. We propose the following homogeneous LF candidate:

$$
\mathscr{V}(x, z)=\theta W^{\alpha}(x, z)-x_{n} z, \alpha=\frac{2+(2 n-1) v}{2(1+n v)}, \theta>0 .
$$

Due to homogeneity we can conclude that $\mathscr{V}$ is positive definite for $\theta>0$ sufficiently large. Its derivative is

$$
\begin{aligned}
\dot{\mathscr{V}}(x, z, d)= & \alpha \theta W^{\alpha-1}\left(-\varepsilon V^{2(1+n v)+v}+\frac{2}{\gamma} z d\right)- \\
& \left(u_{1}(x)+z\right) z-x_{n}\left(u_{2}(x)+d\right) \\
= & -\mathscr{W}(x, z)+\left(\frac{2}{\gamma} \alpha \theta W^{\alpha-1} z-x_{n}\right) d .
\end{aligned}
$$

where

$$
\mathscr{W}(x, z)=\theta \alpha \varepsilon W^{\alpha-1} V^{2(1+n v)+v}+x_{n} u_{2}(x)+u_{1}(x) z+z^{2} .
$$

Note that $\mathscr{V}(x, z)$ is homogeneous of degree $\delta_{\mathscr{V}}=2+$ $(2 n-1) v, \mathscr{W}(x, z)$ is homogeneous of degree $\delta_{\mathscr{W}}=\delta_{\mathscr{V}}+v$, 
while function $\left(\frac{2}{\gamma} \alpha \theta W^{\alpha-1} z-x_{n}\right)$ is homogeneous of degree $\delta_{d}=1+(n-1) v$. The latter two are equal in case $\delta_{\mathscr{V}}+v=$ $\delta_{d}$, that is when $v=-\frac{1}{n+1}$, i.e. when $u_{2}(x)$ is a discontinuous function of homogeneity degree 0 .

Lemma 3: $\mathscr{W}(x, z)>0$ for $\theta>0$ large enough.

Proof: Due to homogeneity and positive definiteness

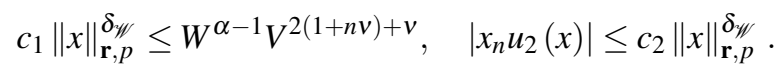

Using Young's inequality we also conclude that

$$
\left|u_{1}(x) z\right| \leq \frac{1}{2} c_{3}\left|u_{1}(x)\right|^{2}+\frac{1}{2 c_{3}} z^{2} \leq \frac{1}{2} c_{3} c_{4}\|x\|_{\mathbf{r}, p}^{\delta_{\mathscr{W}}}+\frac{1}{2 c_{3}} z^{2},
$$

for every $c_{3}>0$ and therefore

$$
\begin{aligned}
\mathscr{W}(x, z) & \geq \theta \alpha \varepsilon c_{1}\|x\|_{\mathbf{r}, p}^{\delta_{\mathscr{W}}}-\left|x_{n} u_{2}(x)\right|-\left|u_{1}(x) z\right|+z^{2} \\
& \geq\left(\theta \alpha \varepsilon c_{1}-c_{2}-\frac{1}{2} c_{3} c_{4}\right)\|x\|_{\mathbf{r}, p}^{\delta_{\mathscr{W}}}+\left(1-\frac{1}{2 c_{3}}\right) z^{2}
\end{aligned}
$$

which is positive definite if we select $1-\frac{1}{2 c_{3}}>0$ and $\theta>0$ sufficiently large.

In any case there exist positive constants $0<\underline{\eta}_{\mathscr{W}}<\bar{\eta}_{\mathscr{W}}$ and $\eta_{d}>0$ such that

$$
\begin{gathered}
\underline{\eta}_{\mathscr{W}} \mathscr{V}^{\frac{\delta_{\mathscr{V}}+v}{\delta_{\mathscr{V}}}}(x, z) \leq \mathscr{W}(x, z) \leq \bar{\eta}_{\mathscr{W}} \mathscr{V}^{\frac{\delta_{\mathscr{V}}+v}{\delta_{\mathscr{V}}}}(x, z) \\
\left|\frac{2}{\gamma} \alpha \theta W^{\alpha-1} z-x_{n}\right| \leq \eta_{d} \mathscr{W}^{\frac{\delta_{d}}{\delta_{\mathscr{V}}+v}}(x, z) \leq \eta_{d} \bar{\eta}_{\mathscr{W}}^{\frac{\delta_{d}}{\delta_{\mathscr{W}}+v}} \mathscr{V}^{\frac{\delta_{d}}{\delta_{\mathscr{V}}}}(x, z),
\end{gathered}
$$

and thus the inequality (14) can be written as

$$
\dot{\mathscr{V}}(x, z, d) \leq-\underline{\eta}_{\mathscr{W}} \mathscr{\mathscr { V }}^{\frac{\delta_{\mathscr{V}}+v}{\delta_{\mathscr{V}}}}(x, z)+\eta_{d} \bar{\eta}_{\mathscr{W}}^{\frac{\delta_{d}}{\delta_{\mathscr{W}}+v}} \mathscr{V}^{\frac{\delta_{d}}{\delta_{\mathscr{V}}}}(x, z) d .
$$

From inequality (15) we obtain the following conclusions:

1) When $d \equiv 0$ the origin $(x, z)=0$ is exponentially stable for $v=0$ or finite time stable otherwise.

2) When $d \neq 0$ and $|d| \leq D$ we have two situations:

a) If $-\frac{1}{n+1}<v \leq 0$ we conclude that the system is ISS with respect to $d$.

b) If $v=-\frac{1}{n+1}$ then we conclude that, for given values of gains, there is a value $D>0$ for which the origin $(x, z)=0$ is finite time stable.

\section{Simulation Results}

Consider the following chain of integrators

$$
\dot{x}_{1}=x_{2}, \quad \dot{x}_{2}=x_{3}, \quad \dot{x}_{3}=u+\rho .
$$

We use for the simulations the Runge-Kutta's integration method with a sampling time of $1 \times 10^{-3}$ [s]. The perturbation is given by $\rho=1+0.1 \sin (t)$, which consists of a constant and a time-varying term, that has a Lipschitz constant $D=$ 0.1 . The initial condition is $x_{0}=x(0)=\left[\begin{array}{lll}1 & 0 & 0\end{array}\right]^{T}$.

We consider the controller (7) with $\gamma=1, \varepsilon=1$ for three homogeneity degrees $v=\left\{0,-\frac{1}{8},-\frac{1}{4}\right\}$ and, solving the equations (6) using Yalmip of Matlab, we obtain

$$
\begin{aligned}
P_{L}=\left[\begin{array}{lll}
5.7359 & 4.5486 & 0.9842 \\
4.5486 & 5.0265 & 1.2232 \\
0.9842 & 1.2232 & 0.6116
\end{array}\right], & K_{L}^{T}=\left[\begin{array}{l}
5.8278 \\
7.8278 \\
3.0000
\end{array}\right], \\
P_{H}=\left[\begin{array}{lll}
5.2763 & 3.9664 & 0.8775 \\
3.9664 & 4.1913 & 0.9975 \\
0.8775 & 0.9975 & 0.5320
\end{array}\right], & K_{H}^{T}=\left[\begin{array}{l}
6.0126 \\
7.6532 \\
2.6250
\end{array}\right], \\
P_{D}=\left[\begin{array}{lll}
4.6585 & 3.2047 & 0.6911 \\
3.2047 & 3.2157 & 0.6974 \\
0.6911 & 0.6974 & 0.3985
\end{array}\right], & K_{D}^{T}=\left[\begin{array}{l}
6.7412 \\
8.0537 \\
2.2500
\end{array}\right],
\end{aligned}
$$

where $L$ represents the lineal integral controller (with homogeneity degree $v=0), H$ represents the homogeneous continuous one (with homogeneity degree $v=-\frac{1}{8}$ ) and $D$ corresponds to discontinuous case (with homogeneity degree $\left.v=-\frac{1}{4}\right)$. The value of the Lyapunov function is obtained numerically on-line using the method presented in [6].

In Figure 1, the behavior of the state $x_{1}$ is presented. All controllers are able to compensate the constant term of the perturbation, but only the discontinuous integral controller is able to also eliminate the effect of the time-varying term. The continuous controllers (with $v=0,-\frac{1}{8}$ ) can only attenuate the effect of the sinus perturbation term, being the attenuation effect of the nonlinear homogeneous controller stronger.

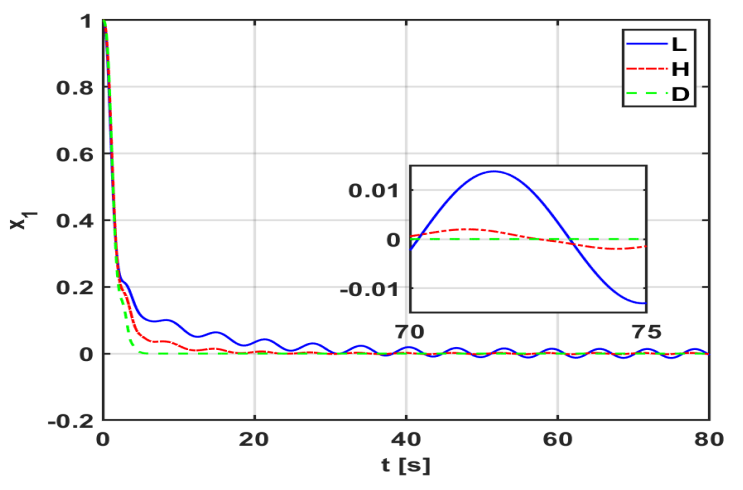

Fig. 1. Time evolution of state $x_{1}$.

Figure 2 shows the behavior of the control input $u$. Note first that it is in all cases a continuous signal. All fluctuate around a constant value, since all controllers compensate the constant term of the perturbation. Moreover, since the discontinuous controller compensates fully the perturbation, the control input contains the perturbation signal, but this is not exactly the case for the continuous controllers. This is confirmed by the Figure 3, that shows the signal $z+\rho$, that can be interpreted as the integral error, since it becomes zero when the integral action completely compensates the perturbation. In the figure we see that for the discontinuous controller the integral error becomes zero after a finite time, while for the continuous controllers the compensation is not perfect, and thus this signal does not converge to zero.

\section{CONCLUSIONS}

We have presented a method to design homogeneous integral controllers for uncertain systems in the normal form, 


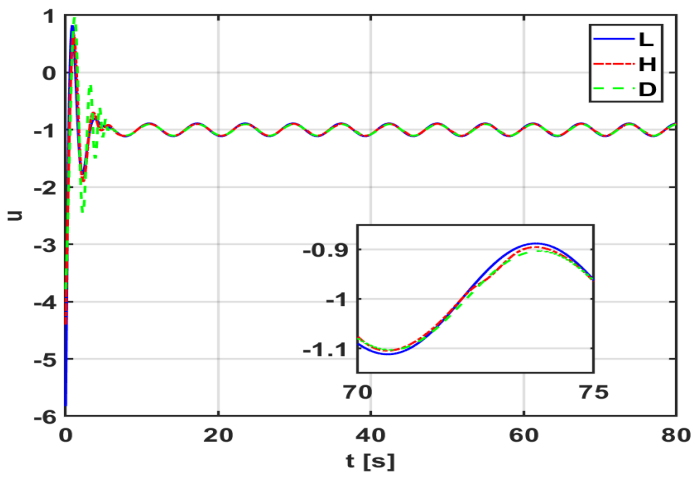

Fig. 2. Time evolution of the control Signal $u(t)$.

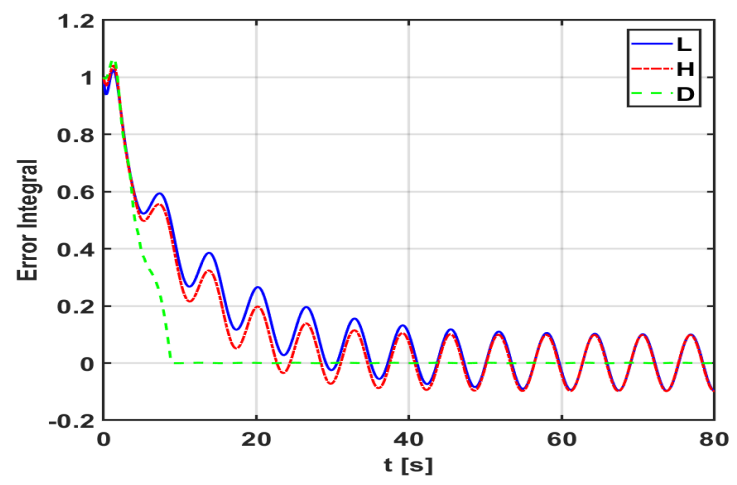

Fig. 3. Integral Error

using the Implicit Lyapunov Function (ILF) approach. The integral controller consists of a homogeneous continuous state feedback and a homogeneous (possibly discontinuous) integral action. The state feedback is constructed with the Implicit Lyapunov Function approach, and the integral term is derived from an explicit strong Lyapunov function based on the ILF. Our method is complementary to other existing approaches to design integral controllers, and it has the advantage that the selection of the gains is reduced to a related problem for linear systems, leading to LMI's. Also the proof becomes rather transparent.

Integral controllers are a fantastic tool to compensate for non vanishing perturbations using continuous control signals, and thus avoiding the discontinuous control signals produced by High Order Sliding Modes, that lead to a strong chattering effect. It is shown that integral controllers with discontinuous control action are able to fully compensate Lipschitz perturbations.

\section{ACKNOWLEDGEMENTS}

The authors would like to thank the financial support from PAPIIT-UNAM (Programa de Apoyo a Proyectos de Investigación e Innovación Tecnológica), project IN110719; CONACyT (Consejo Nacional de Ciencia y Tecnología) CVU 705765. The results on control design using ILF presented in Section $\mathrm{V}$ were developed under support of RSF (grant 17-19-01422) in ITMO University.

\section{REFERENCES}

[1] A. Isidori, Nonlinear control systems, 3rd ed. Berlin: Springer Verlag, 1995.

[2] V. Utkin, Sliding Modes in Control and Optimization. Berlin: Springer-Verlag, 1992.

[3] L. Fridman and A. Levant, Sliding mode in control engineering. Marcel Dekker, Inc. High Order Siliding Modes, 2002.

[4] A. Levant, "Quasi-continuous high-order sliding-mode controllers," IEEE Transactions on Automatic Control, vol. 50, pp. 1812-1816, November 2005.

[5] E. Cruz-Zavala and J. Moreno, "Homogeneous high order sliding mode design: a Lyapunov approach," Automatica, vol. 80, pp. 232238, 2017.

[6] A. Polyakov, D. Efimov, and W. Perruquetti, "Finite-time and fixedtime stabilization: Implicit Lyapunov function method," Automatica, vol. 51, no. 1, pp. 332-340, 2015.

[7] H. K. Khalil, Nonlinear systems. Prentice-Hall, 3rd ed., 2002.

[8] A. Levant., "Homogeneity approach to high-order sliding mode," Automatica, vol. 41, pp. 823-830, 2005.

[9] J. Moreno and M. Osorio, "A Lyapunov approach to second-order sliding mode controllers and observers," in Proceedings of the IEEE Conference on Decision and Control, 2008, pp. 2856-2861.

[10] H. Nakamura, N. Nishida, and N. Nakamura, "High precision control of robots manipulators via finite-time p-pi control," in 51st IEEE Conference on Decision and Control, Japan, December 2012.

[11] C. Zamora, J. A. Moreno, and S. Kamal, "Control integral discontinuo para sistemas mecánicos," in 2013 Congreso Nacional de Control Automático (CNCA AMCA). Ensenada, Baja California, Mexico: Asociación de México de Control Automático (AMCA), Oct 2013, pp. 11-16. [Online]. Available: http://amca.mx/memorias/amca2013/articulos/0155.pdf

[12] J. A. Moreno, "Discontinuous integral control for mechanical systems," in 14th International Workshop on Variable Structure Systems (VSS), Nanjing, 2016, pp. 142-147.

[13] S. Kamal, J. Moreno, A. Chalanga, B. Bandyopadhyay, and L. Fridman, "Continuous terminal sliding-mode controller," Automatica, vol. 69 , pp. 308-314, 2016.

[14] A. Mercado-Uribe and J. A. Moreno, "Discontinuous integral control for systems in controller form," in Congreso Nacional de Control Automtico 2017. Mexico: Asociación de México de Control Automático (AMCA), October 2017. [Online]. Available: http://cnca2017.fime.uanl.mx/MemoriaCNCA17/media/files/0017.pdf

[15] J. A. Moreno, Discontinuous Integral Control for Systems with relative degree two. Chapter 8 In: Julio Clempner, Wen Yu (Eds.), New Perspectives and Applications of Modern Control Theory;in Honor of Alexander S. Poznyak. Springer International Publishing, 2018.

[16] S. Laghrouche, M. Harmouche, and Y. Chitour, "Higher order supertwisting for perturbed chains of integrators," IEEE Transactions on Automatic Control, vol. 62, no. 7, pp. 3588-3593, July 2017.

[17] E. Bernuau, D. Efimov, W. Perruquetti, and A. Polyakov, "On homogeneity and its application in sliding mode control," Journal of the Franklin Institute, vol. 351, no. 4, pp. 1816-1901, 2014.

[18] A. Baccioti and L. Rosier, Lyapunov functions and stability in control theory. New York: Springer-Verlag, $2^{\text {nd }}$ ed., 2005.

[19] L. Rosier, "Homogeneous Lyapunov function for homogeneous continuous vector field," Systems \& Control Letters, vol. 19, no. 6, pp. 467-473, 1992

[20] H. Nakamura, Y. Yamashita, and H. Nishitani, "Smooth lyapunov functions for homogeneous differential inclusions," in In Proceedings of the 41st SICE Annual Conference, vol. 3, Japan, December 2002, p. 19741979.

[21] S. Bhat and D. Bernstein, "Geometric homogeneity with applications to finite-time stability," Mathematics of Control, Signals, and Systems, vol. 17, no. 2, pp. 101-127, 2005.

[22] E. Bernuau, D. Efimov, W. Perruquetti, and A. Polyakov, "On an extension of homogeneity notion for differential inclusions," in European Control Conference, Zurich, Switzerland, July 2013.

[23] A. Filippov, Differential equations with discontinuous righthand side. Kluwer. Dordrecht, The Netherlands, 1988.

[24] D. Gutierrez-Oribio, A. Mercado-Uribe, J. Moreno, and L. Fridman, "Stabilization of the reaction wheel pendulum via a third order discontinuous integral sliding mode algorithm," in Proceedings of IEEE International Workshop on Variable Structure Systems, July 2018, pp. 132-137. 